\title{
Cardiac Tamponade: A Rare Manifestation of Familial Mediterranean Fever
}

\author{
Abdolreza Malek $\mathbb{D}^{\mathrm{D}},{ }^{1}$ Tina Zeraati $\mathbb{D}^{\mathrm{D}},{ }^{2,3}$ Ariane Sadr-Nabavi $\mathbb{D}^{\mathrm{D}},{ }^{2,4,5,6,7}$ Niloofar Vakili ${ }^{\mathbb{D}}{ }^{1}$ \\ and Mohammad Reza Abbaszadegan $\mathbb{D D}^{2,4}$ \\ ${ }^{1}$ Department of Pediatrics, Faculty of Medicine, Mashhad University of Medical Sciences, Mashhad, Iran \\ ${ }^{2}$ Medical Genetics Research Center, Mashhad University of Medical Sciences, Mashhad, Iran \\ ${ }^{3}$ Kidney Transplantation Complications Research Center, Mashhad University of Medical Sciences, Mashhad, Iran \\ ${ }^{4}$ Department of Medical Genetics \& Molecular Medicine, Faculty of Medicine, Mashhad University of Medical Sciences, \\ Mashhad, Iran \\ ${ }^{5}$ Academic Center for Education, Culture, and Research (ACECR)-Khorasan Razavi, Mashhad, Iran \\ ${ }^{6}$ Institute of Neurogenomics, Helmholtz Zentrum München, Munich, Germany \\ ${ }^{7}$ Institut für Humangenetik, Technische Universität München, Munich, Germany
}

Correspondence should be addressed to Mohammad Reza Abbaszadegan; abbaszadeganmr@mums.ac.ir

Received 3 October 2021; Revised 22 December 2021; Accepted 29 December 2021; Published 7 February 2022

Academic Editor: Gregory J. Tsay

Copyright (c) 2022 Abdolreza Malek et al. This is an open access article distributed under the Creative Commons Attribution License, which permits unrestricted use, distribution, and reproduction in any medium, provided the original work is properly cited.

Familial Mediterranean fever (FMF) typically presents with recurrent attacks of fever and serosal inflammation with peritoneum, pleura, and synovium. We usually do not expect pericardial involvement at the early stages. FMF is an autoinflammatory disease, usually inherited with an autosomal recessive pattern. The patients typically have biallelic mutations in the MEFV gene, located on chromosome 16. Colchicine is the first-line treatment of FMF, which not only plays a crucial prophylactic role regarding the attack episodes, but also prevents amyloidosis. Colchicine resistance and intolerance in FMF patients have been rarely reported. Alternative anti-inflammatory agents are understood to be helpful in such cases. We describe a 13-year-old boy referred to our pediatric department complaining of chest pain, dyspnea, and tachycardia. Due to the massive pericardial and pleural effusion, a pericardiocentesis was performed, and a chest tube was inserted. Cardiac tamponade was considered as the initial diagnosis. After a month, he faced another episode of pleuritic chest pain, fever, tachycardia, and pleural and pericardial effusion. Evaluation for probable differential diagnoses including infection, malignancy, and collagen vascular disease showed no remarkable results. Finally, the mutation found by whole exome sequencing was confirmed by direct Sanger sequencing revealing a heterozygote c.44G > C (p.Glu148Gln) mutation in exon 2, confirming the clinical diagnosis of familial Mediterranean fever. Since he seemed to be nonresponsive to the maximum standard dose of colchicine, $100 \mathrm{mg}$ of daily dapsone was added to his treatment regimen, which controlled the attack episodes well. FMF, while rarely initiated with cardiac manifestation, should be considered in patients with any early signs and symptoms of cardiovascular involvement.

\section{Introduction}

Familial Mediterranean fever (FMF) is an autoinflammatory disease, typically presented with recurrent fever episodes in addition to serosal inflammation symptoms. However, cardiac involvement mainly occurs in the advanced stages of the disease, primarily due to the growing inflammation and sedimentation of amyloid in the vessels. Basically, the condition is rarely initiated by cardiac symptoms [1].

Colchicine is the treatment of choice for FMF patients; however, there have been reported cases from different parts of the world whose disease was not controlled only by 
colchicine. Dapsone is considered to be added to colchicine for those not responding to the standard colchicine therapy and those who cannot tolerate higher doses of colchicine [2]. The accurate definition of colchicine resistance in FMF patients has not been well established. Various factors have been considered in the literature, including attack frequency, attack duration, physician assessment, clinical symptoms, and the level of acute phase reactants.

We hereby describe a case of FMF diagnosed based on the clinical symptoms and the findings of direct Sanger sequencing. Severe presentation is not expected in FMF patients with exon 2 mutations; however, our case was presented with pericarditis and pleurisy for the first time. Moreover, the attacks were not controlled prior to the addition of Dapsone to the colchicine regimen.

\section{Case Presentation}

A 13-year-old boy presented to Imam Reza hospital in Mashhad with chest pain and dyspnea. His parents denied any remarkable past medical history but mentioned mild influenza a few days before admission. His parents were not relatives, and his family history included some cases of arthritis and autoimmune diseases among his paternal cousins. A sepsis workup and a cardiopulmonary assessment were initiated. Labs were positive for an elevated sedimentation rate (ESR) and an elevated C-reactive protein, in addition to mild anemia (Table 1). Cardiomegaly was observed in the chest X-ray. A bilateral pleural effusion, dominant in the right side, was found through chest ultrasound. Furthermore, the presence of fluid with a $25 \mathrm{~mm}$ diameter around his heart was confirmed. Massive pericardial effusion was considered based on the findings of echocardiography. The electrocardiogram showed alternans cardiac waves; however, cardiac biomarkers were negative. As his signs and symptoms were strongly suggestive for cardiac tamponade, pericardiocentesis was performed, and a chest tube was inserted concerning pleural effusion. Subacute fibrinous pericarditis associated with reactive mesothelial hyperplasia was observed through the histologic assessment of the pericardium. The pericardial fluid analysis and culture were not suggestive of neither malignancy nor infection. Finally, he was discharged and placed on oral clindamycin (600 mg every 6 hours) and cefixime ( $400 \mathrm{mg}$ once a day).

After a month, he was referred for the second time, complaining of fever, tachycardia, and pleuritic chest pain. Parahilar haziness and peribronchial thickening were seen in his chest X-ray. The chest ultrasound showed left-sided pleural effusion, and it confirmed the presence of fluid around the heart with a $7 \mathrm{~mm}$ diameter. His cardiac biomarkers were negative. Laboratory results were significant for leukocytosis $13.34 * 10 \hat{3} / \mathrm{uL}$ and an ESR of $57 \mathrm{~mm} / \mathrm{hr}$. Blood culture, urine culture, and serologic tests were negative (Table 1). The patient fulfilled major Tel-Hashomer criteria for FMF (recurrent febrile episodes accompanied by pleurisy). Besides, he fulfilled one major and two minor Livneh FMF criteria, including unilateral pleuritis or pericarditis accompanied by severe attacks requiring bed rest; at
TABLE 1: Laboratory results at first and second admission.

\begin{tabular}{|c|c|c|}
\hline Laboratory tests & First admission & Second admission \\
\hline Urea & Not determined & $25 \mathrm{mg} / \mathrm{dl}$ \\
\hline $\mathrm{Cr}$ & Not determined & $0.8 \mathrm{mg} / \mathrm{dl}$ \\
\hline Uric acid & Not determined & $6.7 \mathrm{mg} / \mathrm{dl}$ \\
\hline $\mathrm{Na}$ & Not determined & $139-137 \mathrm{mEq} / \mathrm{L}$ \\
\hline $\mathrm{K}$ & Not determined & $3.5-4 \mathrm{mEq} / \mathrm{L}$ \\
\hline AST & Not determined & $18 \mathrm{U} / \mathrm{L}$ \\
\hline ALT & Not determined & $22 \mathrm{U} / \mathrm{L}$ \\
\hline $\mathrm{LDH}$ & Not determined & $376 \mathrm{U} / \mathrm{L}$ \\
\hline CPK & Not determined & 37 \\
\hline CK-MB & 12 & 9 \\
\hline TPI & - & - \\
\hline ESR & 11 & 57 \\
\hline CRP & 37.9 & Not determined \\
\hline TSH & $6 \mu \mathrm{U} / \mathrm{dl}$ & \\
\hline $\mathrm{T} 4$ & $0.8 \mu \mathrm{g} / \mathrm{dl}$ & \\
\hline ASO & Not determined & 100 \\
\hline Ferritin & Not determined & $317 \mathrm{ng} / \mathrm{mL}$ \\
\hline \multirow{4}{*}{ VBG } & Not determined & 7.329 \\
\hline & Not determined & 18.9 \\
\hline & Not determined & 34.4 \\
\hline & Not determined & 30 \\
\hline O2SAT & Not determined & 53.4 \\
\hline \multirow[t]{2}{*}{ ACE } & Not determined & \\
\hline & $9.2 * 10^{3}$ microliter & 13.34 \\
\hline PMN & $77.9 \%$ & $75 \%$ \\
\hline \multirow{2}{*}{$\mathrm{CBC}$} & $14.1 \%$ & $20 \%$ \\
\hline & $12.3 \mathrm{~g} / \mathrm{dl}$ & 12.1 \\
\hline PLT & $334 * 10^{3}$ microliter & 203 \\
\hline Wright & Not determined & - \\
\hline Coombs wright & Not determined & - \\
\hline $2 \mathrm{me}$ & Not determined & - \\
\hline Widal & Not determined & - \\
\hline ANA & Not determined & - \\
\hline EBV IgM & Not determined & - \\
\hline $\mathrm{C} 4$ & Not determined & - \\
\hline $\mathrm{C} 3$ & Not determined & - \\
\hline CH50 & Not determined & - \\
\hline $\mathrm{UC}$ & Not determined & - \\
\hline $\mathrm{BC}$ & - & - \\
\hline
\end{tabular}

the same time, there were some attack-free intervals, and his age was below 20 years [3]. Therefore, following whole exome sequencing, direct Sanger sequencing was performed to confirm the mutation and the diagnosis of FMF. The mutation, c.44G > C (p.Glu148Gln), was detected in exon 2 of the MEFV-gene in the heterozygote state, which is typically associated with FMF. Hence, he was placed on $1 \mathrm{mg}$ of daily colchicine, but it did not seem to be effective. After six months, the colchicine dose was increased to $2 \mathrm{mg}$ per day, which resulted in significant symptom improvement during the following six months. However, he faced the side effects of colchicine, such as an increase of liver enzyme levels. Tapering the colchicine dose resulted in the recurrence of disease attacks and an increased rate of weekly hospitalization. The clinical manifestation and essential laboratory data, including WBC, ESR, CRP, and PLT during hospitalization in different episodes and the interval between the episodes, are recorded (Table 2). Apparently, the serum level of acute phase reactants was determined within the normal 
TABLE 2: Clinical and laboratory findings during episodes and between the episodes.

\begin{tabular}{|c|c|c|c|c|c|c|c|}
\hline & Episode 1 & Episode 2 & Episode 3 & Episode 4 & Episode 5 & $\begin{array}{l}\text { Between episodes } \\
\text { (without attack) }\end{array}$ & $\begin{array}{l}\text { The latest } \\
\text { checkup }\end{array}$ \\
\hline $\begin{array}{l}\text { Clinical } \\
\text { manifestation }\end{array}$ & $\begin{array}{c}\text { Weakness, } \\
\text { pleuritic chest } \\
\text { pain }\end{array}$ & $\begin{array}{l}\text { Abdominal } \\
\text { pain }\end{array}$ & $\begin{array}{l}\text { Abdominal } \\
\text { pain }\end{array}$ & $\begin{array}{l}\text { Fever, chest } \\
\text { pain }\end{array}$ & $\begin{array}{c}\text { Fever, chest } \\
\text { pain }\end{array}$ & No symptoms & No symptoms \\
\hline WBC & $\begin{array}{c}12.76 * 10^{3} \\
\text { microliter }\end{array}$ & $\begin{array}{l}15.9 * 10^{3} \\
\text { microliter }\end{array}$ & $\begin{array}{l}9.19 * 10^{3} \\
\text { microliter }\end{array}$ & $\begin{array}{l}8.52 * 10^{3} \\
\text { microliter }\end{array}$ & $\begin{array}{l}8.91 * 10^{3} \\
\text { microliter }\end{array}$ & $\begin{array}{l}12.6 * 10^{3} \\
\text { microliter }\end{array}$ & $\begin{array}{c}5 * 10^{3} \\
\text { microliter }\end{array}$ \\
\hline ESR & 5 & 52 & 7 & 24 & 99 & 3 & 3 \\
\hline CRP & 44.61 & 83 & 15.1 & 223 & 200 & 2 & $\begin{array}{c}\text { Not } \\
\text { determined }\end{array}$ \\
\hline PLT & $\begin{array}{l}209 * 10^{3} \\
\text { microliter }\end{array}$ & $\begin{array}{l}284 * 10^{3} \\
\text { microliter }\end{array}$ & $\begin{array}{l}190 * 10^{3} \\
\text { microliter }\end{array}$ & $\begin{array}{l}197 * 10^{3} \\
\text { microliter }\end{array}$ & $\begin{array}{l}288 * 10^{3} \\
\text { microliter }\end{array}$ & $\begin{array}{l}187 * 10^{3} \\
\text { microliter }\end{array}$ & $\begin{array}{l}193 * 10^{3} \\
\text { microliter }\end{array}$ \\
\hline
\end{tabular}

$\mathrm{Cr}$, creatinine; AST, aspartate transaminase; ALT, alanine transaminase; LDH, lactate dehydrogenase; $\mathrm{CPK}$, creatine kinase; CK-MB, creatine kinase-MB; TPI, troponin; ESR, erythrocyte sedimentation rate; CRP, C-reactive protein; TSH, thyroid-stimulating hormone; ASO, antistreptolysin O; VBG, venous blood gas; ACE, angiotensin-converting enzyme; CBC, complete blood count; WBC, white blood cells; PMN, polymorphonuclear leukocytes; lymph, lymphomononuclear cells; $\mathrm{Hb}$, hemoglobin; PLT, platelet count; 2ME, 2-mercaptoethanol; ANA, antinuclear antibodies; EBV IgM, Epstein-Barr virus antibody; UC, urine culture; $\mathrm{BC}$, blood culture.

range whenever the patient had no symptoms. After six months, $100 \mathrm{mg}$ of daily dapsone was added to his treatment regimen. It was so effective that he experienced only one attack in two years.

\section{Discussion}

Familial Mediterranean fever is an autoimmune disease resulting from mutations in the MEFV gene, which encodes pyrin, an inflammasome protein [4]. The gene is located on chromosome 16, and the mutations in this gene are accompanied by both autosomal recessive and autosomal dominant patterns of inheritance. The mutation inherited in an autosomal recessive pattern is usually considered homozygous or compound heterozygous, while the mutation inherited by an autosomal dominant pattern is heterozygous. Most of the identified mutations are located on exon 10 , associated with more severe disease, in addition to several mutations on exon 2 , which are usually associated with milder symptoms [5]. In 2019, Bilge et al. [6] conducted a study to compare the disease severity of FMF patients with identified mutations located in exon 10 and exon 2. Their findings suggested that amyloidosis, arthritis, and erysipelaslike erythema (ELE) were more frequent in patients with a mutation in exon 10. However, the other symptoms, including peritonitis, fever, pleuritis, vasculitis, myalgia, and chronic renal failure (CRF), were similarly frequent in both groups.

In 2018, Barut et al. [7] conducted a study evaluating the demographic characteristics, clinical manifestations, gene mutations, and responses to the treatment of 708 FMF patients. They reported that the $\mathrm{M} 694 \mathrm{~V}$ mutation, responsible for the most symptoms, is the most frequent mutation in FMF patients. The gene is expressed in $63.8 \%$ of patients who are nonresponsive to colchicine, while only $17 \%$ of complete colchicine responders have the mutation.

In 2017, Yoldas et al. [8] from Turkey reported two cases of massive pericardial effusion, later diagnosed with FMF according to clinical and laboratory findings. Their first case was a 13-year-old girl with chest pain, dyspnea, fever, tachycardia, and distant heart sounds. Laboratory findings confirmed a low level of hemoglobin (Hb: $9.7 \mathrm{mg} / \mathrm{dl}$ ) and an increased level of ESR and CRP without leukocytosis. She was diagnosed with purulent pericarditis due to the accumulation of exudative pericardial fluid; hence, the antibiotic treatment was applied for her. In spite of the appropriate treatment, the amount of pericardial fluid increased massively in four days. The evaluation for connective tissue disorders showed heterozygous R202Q, A165A, G138G, and D102D mutations in exon 2, suggestive for FMF [7]. The second case was a 10-year-old boy referred to the emergency department complaining of chest pain, dyspnea, and cough for two weeks. Muffled heart sounds, tachycardia, and tachypnea were detected through physical examination. Laboratory studies revealed an increased level of acute-phase reactants. Massive pericardial effusion was confirmed by echocardiography, and one liter of hemorrhagic pericardial fluid was drained. She was initially diagnosed with purulent pericarditis and was placed on broad-spectrum antibiotics, but the pericardial fluid culture was negative. Finally, FMF gene analysis revealed homozygous D102D, G138G, A165A, and R202Q mutations in exon 2. Since colchicine treatment failed to reduce the pericardial effusion and stop FMF attack episodes, naproxen was added to his treatment regimen, followed by an acceptable treatment response.

A similarity between the Turkish reported case [8] and our case is that they both manifested cardiac symptoms, in addition to colchicine resistance. As Soriano et al. claimed in their review article, published in 2020, the benefits of colchicine treatment were described in 1972 for the first time. Its efficacy and potential to prevent FMF attack episodes have been proven through numerous clinical trials since then [9]. The total dose of oral colchicine in children with FMF is 0.5 to $2 \mathrm{mg}$ per day, which is usually well-tolerated. Yet, some gastrointestinal adverse events and increased levels of transaminases have been reported following colchicine treatment with the therapeutic dose. Besides, approximately $5 \%$ of patients have remained colchicine resistant [10]. For decades, rheumatologists have been establishing fixed criteria to address colchicine resistance 
identically. They have suggested various measures, including Ben-Chetrit et al. [14], Hentgen et al. [15], and Ozen et al. [16] (10). It seems that the most convincing criteria published to date are those suggested by the EULAR (European League Against Rheumatism). According to EULAR, compliant patients receiving the maximum tolerated dose of colchicine for six months are considered colchicine resistant, providing that they experience one or more attacks monthly. An alternative treatment strategy is required for such patients and those who are suspicious of significant subclinical inflammation leading to secondary amyloidosis [10].

The first alternative treatment for colchicine-resistant patients is NSAID. NSAIDs have appeared effective in some FMF cases, most of whom have manifested with myalgia. The other effective anti-inflammatory agents are glucocorticoids. However, these agents are only used in attack episodes in the form of the intravenous methylprednisolone pulse. Thalidomide is another anti-inflammatory agent. It is used in various inflammatory conditions exclusively for dermatological diseases, such as cutaneous lupus erythematosus. In 2006, Turkish rheumatologists added thalidomide and etanercept to the colchicine regimen. The regimen was applied for the colchicine-resistant FMF patients. Four male patients received thalidomide $100 \mathrm{mg} /$ day, replaced with etanercept for 2 of them, due to thalidomide intolerance, and one female patient received etanercept as the first choice due to the expected side effects of thalidomide in future pregnancy. Their results showed that both thalidomide and etanercept might lower abdominal attacks [14]; however, thalidomide is still not considered as an excellent additional therapy for colchicine resistant patients [10]. The other alternative for colchicine is interferon $\alpha$ that can suppress acute inflammation; however, it is less effective than other agents [15].

Another remarkable point is the elevated serum level of specific cytokines such as interleukin-1, interleukin-6, and TNF $\alpha$ in the patients. Thus, anti-interleukin 1 , anti-interleukin 6 , anti-TNF, and Janus kinase inhibitors are expected to be beneficial [16].

Finally, the use of dapsone regarding colchicine resistance is recommended. Dapsone is a sulfonamide known for its both antibacterial and anti-inflammatory features. It is mainly used for neutrophil-dominant dermatologic disorders [17]. It is likely to increase the anti-inflammatory effect when added to colchicine, downregulating neutrophil-mediated inflammatory responses. Inhibiting the production of reactive oxygen species and reducing the effect of eosinophil peroxidase on mast cells are the other anti-inflammatory mechanisms of dapsone [18]. Dapsone is also known for its anti-inflammatory effects regarding a great many inflammatory disorders. For instance, in 2019, Rashidian et al. [19] demonstrated the anti-inflammatory effects of dapsone on acetic acid-induced colitis in rats, probably through NF-kB pathway inhibition. In 2012, Salehzadeh et al. in Iran added $2 \mathrm{mg} / \mathrm{kg}$ of dapsone to the standard colchicine-based regimen of $10 \mathrm{FMF}$ patients who were nonresponsive to colchicine; the follow-up duration was six months. The results of their study demonstrated that dapsone appears to control attack episodes of FMF in patients who are nonresponsive to colchicine [2]. Dapsone is also inexpensive and available, and it has fewer side effects than other alternative drug choices. Providing we add it to the standard dose of colchicine in colchicineresistant patients, the attack rate is expected to decrease.

\section{Conclusion}

Familial Mediterranean fever is not usually manifested with cardiac symptoms for the first time. Cardiac involvement usually occurs at the progressed stages of the disease. Yet, tamponade and pericardial effusion may rarely occur as the first attack episodes of FMF. Early diagnosis and applying the anti-inflammatory treatment in FMF are critical to prevent complications such as amyloidosis. Rheumatologists should be aware of these rare manifestations of FMF, and they ought to seek confirmatory investigations such as whole exome sequencing followed by direct Sanger sequencing confirmation of the mutation before finalizing clinical management to avoid misdiagnosis. Besides, the results of gene assessment can be used for genetic counseling to prevent disease occurrence in the next generation. Furthermore, although colchicine is the treatment of choice in FMF patients, some cases do not respond to the therapeutic doses, and they experience adverse effects with escalating doses. Dapsone seems to be an acceptable alternative which not only reduces the attack episodes but also prevents amyloidosis when it is added to colchicine.

\section{Data Availability}

Considering the patient privacy as an ethical concern, access to data is restricted.

\section{Conflicts of Interest}

The authors declare no conflicts of interest.

\section{Authors' Contributions}

AM and TZ collected the data and drafted the manuscript. AM designed the study. TZ collected the data. AM and MA reviewed the manuscript. NV, AS, and MA contributed to the development of the study. All authors read and signed the final paper.

\section{Acknowledgments}

The authors appreciate the cooperation of the staff of the pediatric ward of Imam Reza and Akbar hospitals in Mashhad, who helped us contact the patient's parents and collect essential data.

\section{References}

[1] A. Alsarah, O. Alsara, and H. S. Laird-Fick, "Cardiac manifestations of familial mediterranean fever," Avicenna journal of medicine, vol. 7, no. 4, pp. 158-163, Article ID 158, 2017.

[2] F. Salehzadeh, S. Jahangiri, and E. Mohammadi, "Dapsone as an alternative therapy in children with familial Mediterranean 
fever," Iranian Journal of Pediatrics (English edition), vol. 22, no. 1, pp. 23-27, 2012.

[3] H. Petrushkin, M. Stanford, F. Fortune, and A. S. Jawad, "Clinical review: familial mediterranean fever-an overview of pathogenesis, symptoms, ocular manifestations, and treatment," Ocular Immunology and Inflammation, vol. 24, no. 4, pp. 422-430, 2016.

[4] Y. H. Park, E. F. Remmers, W. Lee et al., "Ancient familial Mediterranean fever mutations in human pyrin and resistance to Yersinia pestis," Nature Immunology, vol. 21, no. 8, pp. 857-867, 2020.

[5] A. Ece, E. Çakmak, Ü. Uluca et al., "The MEFV mutations and their clinical correlations in children with familial Mediterranean fever in southeast Turkey," Rheumatology International, vol. 34, no. 2, pp. 207-212, 2014.

[6] Ş. Y. Bilge, D. Solmaz, S. Şenel et al., "Exon 2: is it the good police in familial mediterranean fever?" European journal of rheumatology, vol. 6, no. 1, Article ID 34, 2019.

[7] K. Barut, S. Sahin, A. Adrovic et al., "Familial Mediterranean fever in childhood: a single-center experience," Rheumatology International, vol. 38, no. 1, pp. 67-74, 2018.

[8] T. Yoldas, Ș. Kayali, İ. Ertuğrul, V. Doğan, U. Arman Örün, and S. Karademir, "Massive pericardial effusion and tamponade can be a first sign of familial mediterranean fever," Pediatric Emergency Care, vol. 33, no. 9, pp. e48-e51, 2017.

[9] A. Soriano, M Soriano, G Espinosa et al., "Current therapeutic options for the main monogenic autoinflammatory diseases and PFAPA syndrome: evidence-based approach and proposal of a practical guide," Frontiers in Immunology, vol. 11, Article ID 865, 2020.

[10] S. Ozen, I. Kone-Paut, and A. Gül, "Colchicine resistance and intolerance in familial mediterranean fever: definition, causes, and alternative treatments," Seminars in Arthritis and Rheumatism, vol. 47, 2017.

[11] E. Ben-Chetrit and H. Ozdogan, "Non-response to colchicine in FMF--definition, causes and suggested solutions," Clinical and Experimental Rheumatology, vol. 26, no. 4, Suppl 50, pp. S49-S51, 2008.

[12] V. Hentgen, G. Grateau, I. Kone-Paut et al., "Evidence-based recommendations for the practical management of familial mediterranean fever," in Seminars in Arthritis and Rheumatism, vol. 43, no. 3, pp. 387-391, WB Saunders, Philadelphia, PA, USA, 2013.

[13] S. Ozen, I. Kone-Paut, and A. Gül, "Colchicine resistance and intolerance in familial mediterranean fever: definition, causes, and alternative treatments," in Seminars in Arthritis and Rheumatism, vol. 47, no. 1, pp. 115-120, WB Saunders, Philadelphia, PA, USA, 2017.

[14] E. Seyahi, H Ozdogan, S Celik, S Ugurlu, and H Yazici, "Treatment options in colchicine resistant familial Mediterranean fever patients: thalidomide and etanercept as adjunctive agents," Clinical \& Experimental Rheumatology, vol. 24, pp. S99-S103, 2006.

[15] M. Tunca, S Akar, M Soytürk et al., "The effect of interferon alpha administration on acute attacks of familial Mediterranean fever: a double-blind, placebo-controlled trial," Clinical \& Experimental Rheumatology, vol. 22, pp. S37-S40, 2004.

[16] G. El Hasbani, A. Jawad, and I. Uthman, "Update on the management of colchicine resistant familial mediterranean fever (FMF)," Orphanet Journal of Rare Diseases, vol. 14, no. 1, pp. 1-12, 2019.

[17] F. Anzengruber, J. Schenk, V. Graf, T. M. Nordmann, E. Guenova, and R. Dummer, "Dapsone in a large tertiary center: outdated therapeutic option or timeless agent?" Dermatology, vol. 236, no. 3, pp. 183-190, 2020.

[18] N. Ghaoui, E. Hanna, O. Abbas, A. G. Kibbi, and M. Kurban, "Update on the use of dapsone in dermatology," International Journal of Dermatology, vol. 59, no. 7, pp. 787-795, 2020.

[19] A. Rashidian, A. Rashki, A. Abdollahi et al., "Dapsone reduced acetic acid-induced inflammatory response in rat colon tissue through inhibition of NF-kB signaling pathway," Immunopharmacology and Immunotoxicology, vol. 41, no. 6, pp. 607-613, 2019. 